\title{
Essstörungen bleiben ein relevantes Thema für Praxis und Forschung
}

\author{
Ein Interview mit Brunna Tuschen-Caffier
}

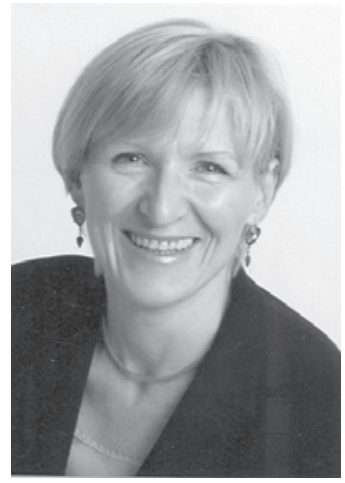

\begin{abstract}
Essstörungen wie die Anorexia nervosa, Bulimia nervosa und Binge-Eating-Störung sind psychische Störungen, die bereits frühzeitig beginnen, gravierende körperliche und psychische Folgeprobleme nach sich ziehen können und häufig einen chronischen Verlauf haben. Daher stellen diese Störungen ein wichtiges Behandlungsfeld für Psychotherapeutinnen und Psychotherapeuten dar. Die häufig zu beobachtende Persistenz der Symptomatik verlangt jedoch nicht nur von den Betroffenen, sondern auch von den Therapeutinnen und Therapeuten eine hohe Frustrationstoleranz. Das Interview mit Frau Prof. Dr. Brunna Tuschen-Caffier, einer in der Forschung exzellent ausgewiesenen "Essstörungsspezialistin" und in der praktischen Umsetzung der kognitiven Verhaltenstherapie erfahrenen Expertin, gibt wichtige Hinweise auf aktuelle Forschungstrends und neue Behandlungsmöglichkeiten im Bereich der verschiedenen Essstörungen. Das Gespräch führte Prof. rer. nat. Ulrike Ehlert, Zürich.
\end{abstract}

Wie groß ist das Risiko, dass sich als Konsequenz von gezügeltem Essverhalten bzw. Diäthalten eine Essstörung ausbildet? Gibt es Prädiktoren einer möglichen Dekompensation?

Auf der Basis retrospektiver Erhebungen ergeben sich Hinweise darauf, dass ein übermäßig ausgeprägtes restriktives Essverhalten sowie extremes Schlankheitsstreben der Entwicklung von Essstörungen vorausgehen können. Auch in prospektiven Längsschnittuntersuchungen zeigte sich, dass ein restriktives Essverhalten die Entwicklung von Essstörungssymptomen vorhersagen konnte. Allerdings fehlen bisher Hinweise darauf, dass dies auch für die Ausbildung des Vollbildes einer Essstörung zutrifft.

Bezüglich der Frage nach potenziellen Risikofaktoren für die Entstehung von Essstörungen ist zu sagen, dass es sinnvoll ist, von einem Vulnerabilitäts-Stress-Modell auszugehen, um der Komplexität der Genese von Essstörungen gerecht zu werden. Neben den bereits genannten Faktoren (gezügelter Essstil, Schlankheitsstreben) können familiäre Faktoren (z.B. gezügelter Essstil der Mutter) und psychische Faktoren (z.B. Selbstwertwertprobleme, Sorgen um Gewicht und Figur) sowie psychosoziale Belastungen (z.B. sexueller Missbrauch) an der Entstehung von Essstörungen beteiligt sein.
Wird es im DSM-5 (www.dsm5.org) gravierende Veränderungen im Bereich der Essstörungsklassifikation geben?

Aus meiner Sicht sind die angedachten Änderungsvorschläge nicht wirklich gravierend, sondern es sind überwiegend Änderungen, die in Richtung Präzisierung der Klassifikationskriterien in Anbetracht der Evidenzlage gehen. So wird vorgeschlagen, bei der Klassifikation der Anorexia nervosa darauf zu verzichten, eine konkrete Zahl zur Definition von Untergewicht anzugeben, sondern vielmehr darauf hinzuweisen, dass Untergewicht in Bezug auf Alter, Geschlecht und den Gesundheitszustand der Patientin bzw. des Patienten definiert werden muss. Lediglich im Begleittext des DSM-5 sollen Beispiele für Untergewicht genannt werden, die aber nicht als fixe Größe genutzt werden sollten. Eine etwas weitreichendere Änderung ist der Vorschlag, dass bei der Klassifikation der Anorexia nervosa auf das Kriterium «Ausbleiben der Menstruation» (bzw. Amenorrhö) verzichtet werden sollte. Der Vorschlag erscheint mir sinnvoll, denn es ist in der Tat so, dass das Kriterium wenig nützlich ist, wenn die Patientinnen mit Anorexia nervosa altersbedingt noch keine Menstruation haben oder wenn sie Kontrazeptiva einnehmen - oder wenn es sich um männliche Patienten handelt.

\section{KARGER}

Fax +497614520714

Information@Karger.de

www.karger.com
두 2011 S. Karger GmbH, Freiburg

Accessible online at:

www.karger.com/ver
Prof. Dr. Brunna Tuschen-Caffier

Institut für Psychologie, Abteilung Klinische und Entwicklungspsychologie

Albert-Ludwigs-Universität Freiburg

Engelbergerstr. 41, 79106 Freiburg

Tel. +49 761 203-3014

tuschen@psychologie.uni-freiburg.de 
Für die Klassifikation der Bulimia nervosa ist es bisher erforderlich, dass die Patient(inn)en über einen Zeitraum von 3 Monaten zweimal in der Woche Essanfälle mit anschließenden Kompensationsmaßnehmen (z.B. Erbrechen) haben. Da sich empirisch gezeigt hat, dass es mit Blick auf klinische Merkmale der Störung keinen Unterschied macht, ob das gestörte Essverhalten und die Kompensationsmaßnahmen regelmäßig ein- oder zweimal pro Woche auftreten, wird vorgeschlagen, das Häufigkeitskriterium auf einmal pro Woche (Essanfälle und Kompensationsmaßnahmen) zu reduzieren. Auch dieser Vorschlag erscheint mir sinnvoll, da sonst viele Patient(inn)en das Vollbild der Bulimia nervosa nicht erfüllen, obwohl sie in Bezug auf andere klinische Merkmale (z.B. Leidensdruck, Verlauf der Störung) dem Krankheitsbild entsprechen. Ferner wird vorgeschlagen, die Unterscheidung in Subgruppen (purging or non-purging type) aufzugeben, da es bisher kaum Hinweise darauf gibt, dass diese Subgruppen klinisch sinnvoll sind. Es scheint eher so zu sein, dass sich große Überschneidungen zur BingeEating-Störung ergeben, wenn die Bulimia nervosa als «nonpurging type» klassifiziert wird.

Des Weiteren wird vor dem Hintergrund der empirischen Befunde vorgeschlagen, dass die Binge-Eating-Störung, die bisher nur als Forschungsdiagnose im DSM-IV-Appendix geführt wird, als eigenständige Essstörungsdiagnose aufgenommen werden sollte. Dies ist meines Erachtens richtig, denn es gibt inzwischen Evidenz, dass die Binge-Eating-Störung sich hinreichend von anderen Essstörungen, insbesondere der Bulimia nervosa, abgrenzen lässt und dass es trotz aller Gemeinsamkeiten zwischen den Essstörungen doch auch Unterschiede, z.B. in Bezug auf den Verlauf oder die Prognose zur Veränderung der Problematik durch unterschiedliche psychotherapeutische Ansätze, gibt. Zum Beispiel gibt es Hinweise darauf, dass bei der Binge-Eating-Störung kognitiv-behaviorale Psychotherapie und interpersonelle Therapie gleichermaBen wirkungsvoll sein können, während sich bei der Bulimia nervosa eine deutliche Überlegenheit der kognitiv-behavioralen gegenüber der interpersonellen Therapie ergibt, wenn man bedenkt, dass die interpersonelle Therapie erst im 1-Jahres-Follow-up eine vergleichbar hohe Wirksamkeit erzielt wie die kognitiv-behaviorale Therapie.

Schließlich wird in dem aktuellen Entwurf zum DSM-5 vorgeschlagen, die Gruppe der nicht näher bezeichneten Essstörungen (Eating Disorder Not Otherwise Specified; EDNOS) zu ersetzen durch eine Gruppe von Störungen, die benannt werden soll als «Feeding and Eating Conditions Not Elsewhere Classified». Hier sollen Patient(inn)en mit atypischer Anorexia nervosa oder mit subsyndromaler Bulimia nervosa sowie subsyndromaler Binge-Eating-Störung eingruppierbar sein. Ferner sind die Kategorien «Purging Disorder» (regelmäßiges Erbrechen ohne vorherige Essanfälle) und das «Night Eating Syndrome» (regelmäßiges nächtliches Essen) sowie eine Restkategorie (Other Feeding or Eating Condition Not Elsewhere Classified) vorgesehen.
Ergeben sich Komorbiditäten zwischen einer Essstörung und weiteren psychischen Störungen?

Essstörungen zeigen durchweg eine hohe Komorbidität mit anderen psychischen Störungen. So weisen Patientinnen mit einer Anorexia nervosa häufig eine Komorbidität mit Depressionen, Angststörungen oder Zwangserkrankungen auf. Dies gilt im Wesentlichen auch für die Bulimia nervosa, wobei Zwangsstörungen bei der Bulimia nervosa weniger häufig komorbid vorkommen als bei der Anorexia nervosa.

Es lassen sich bisher aber keine eindeutigen Aussagen darüber treffen, inwieweit komorbide psychische Störungen Ursache oder Folge der Essstörungen sind. So gehen zu etwa 50-60\% der Fälle Angststörungen und affektive Störungen der Essstörung voraus.

Neben den psychischen Störungen kommen auch Persönlichkeitsstörungen häufig komorbid mit Essstörungen vor, beispielsweise Cluster-C-Persönlichkeitsstörungen (z.B. mit Symptomen der Zwanghaftigkeit, Ängstlichkeit und Vermeidungsverhalten in sozialen Situationen) oder Cluster-BPersönlichkeitsstörungen (z.B. Borderline-Persönlichkeitsstörung).

In der letzten Cochrane-Analyse [Hay et et al., 2009] zur Therapie der Essstörungen aus dem Jahr 2009 wird der kognitiven Verhaltenstherapie eine deutliche Wirksamkeit attestiert. Es wird jedoch auch darauf hingewiesen, dass nach wie vor die Fallzahlen der Therapiestudien zu klein seien. Gibt es aktuelle multizentrische Studien, die zu Wirksamkeitsstudien an größeren Fallzahlen führen werden?

Grundsätzlich ist es richtig, dass es größerer Fallzahlen bedarf, damit klinisch relevante und evidenzbasierte Empfehlungen für die Behandlung der Essstörungen formuliert werden können. Daher ist es sehr begrüßenswert, dass es derzeit in Deutschland große Multicenter-Studien gibt, die vom Bundesministerium für Bildung und Forschung gefördert werden und die klinisch relevante Fragen zur differenziellen Wirksamkeit unterschiedlicher Behandlungszugänge bei Essstörungen beantworten helfen (siehe zum Überblick $w w w . e d n e t-$ essstoerungen.de). So geht es z.B. in einer Studie darum, ein psychodynamisches und ein verhaltenstherapeutisches Therapie-Manual im Vergleich zur ambulanten Standardtherapie der Anorexia nervosa bei Erwachsenen zu überprüfen (ANTOP - Anorexia Nervosa Treatment of OutPatient). Dazu werden ca. 250 Patient(inn)en an 9 universitären Zentren behandelt. Dies ist derzeit die weltweit größte Multicenter-Studie zur Behandlung der Anorexia nervosa. In einem weiteren Teilprojekt wird eine tagesklinische Behandlung von Jugendlichen mit Anorexia nervosa verglichen mit einer stationären Standardtherapie. Rund 170 Patienten sollen eingeschlossen werden. Die Vernetzung in der Therapieforschung ist meines Erachtens sehr wichtig, um substanzielle Erkenntnisse über die Wirksamkeit von Psychotherapie und die Ver- 
besserung der Therapieerfolge - gerade bei einer so schwerwiegenden Erkrankung wie der Anorexia nervosa - zu erzielen. Bei der Behandlung der Anorexia nervosa ist die Evidenzlage derzeit im Vergleich zu den anderen Essstörungen immer noch am schlechtesten. Es gibt bisher kaum randomisierte kontrollierte Therapiestudien und kaum Studien, die direkt verschiedene Behandlungszugänge miteinander vergleichen. Insofern ist es gut, dass die großen Therapiestudien jetzt angelaufen sind.

In einer weiteren Teilstudie des EDNET-Forschungsverbundes wird eine Internettherapie mit einer kognitiv-behavioralen Einzeltherapie in Bezug auf die Wirksamkeit bei der Therapie der Binge-Eating-Störung überprüft (INTERBED). An dieser Studie sind 5 Forschungszentren beteiligt, und es sollen 175 Patienten eingeschlossen werden. Aus aktuellen Studien gibt es bereits deutliche Hinweise darauf, dass bei der Behandlung der Binge-Eating-Störung auch strukturierte Selbsthilfeangebote auf der Basis verhaltenstherapeutischer Selbsthilfe-Manuale substanzielle Verbesserungen der Symptomatik nach sich ziehen können. Insofern ist es auch interessant zu überprüfen, ob durch eine geleitete Internettherapie vergleichbare Effekte erzielt werden können wie durch eine «Face-to-Face»-Verhaltenstherapie.

Des Weiteren werden in anderen Teilprojekten Moderatoren und Mediatoren mit Blick auf die Therapie von Essstörungen untersucht sowie genetische und neurobiologische Faktoren der Ätiologie von Essstörungen erforscht.

Zusammenfassend ist festzuhalten, dass es derzeit große Therapiestudien gibt, die sicher substanzielle Erkenntnisse zur Therapie der Essstörungen liefern werden.

Welche therapeutischen Vorgehensweisen gelten derzeit als am erfolgversprechendsten bei den verschiedenen Subgruppen von Essstörungen?

In der S3-Leitlinie, die derzeit in Deutschland als AWMFLeitlinie zur Behandlung von Essstörungen vorbereitet wird, wird empfohlen werden, dass für alle verschiedenen Formen von Essstörungen die Psychotherapie als Behandlungsverfahren der ersten Wahl gilt. Des Weiteren liegt zumeist für die kognitiv-behaviorale Therapie die deutlichste Evidenz vor. Im Einzelnen ergeben sich allerdings doch Unterschiede bezüglich der Evidenzbasierung psychotherapeutischer Verfahren bei den unterschiedlichen Essstörungen. Bei der Bulimia nervosa liegen deutliche Hinweise darauf vor, dass eine kognitivbehaviorale Therapie gegenüber anderen Verfahren am effektivsten ist. So setzt der Behandlungserfolg deutlich schneller ein als z.B. bei der interpersonellen Therapie. Aber letztlich muss auch gesagt werden, dass zwar bei einem hohen Prozentsatz der Patient(inn)en eine deutliche Besserung der Symptomatik durch eine kognitiv-behaviorale Therapie erzielt werden kann, dass aber bei einem deutlich geringeren Prozentsatz eine vollständige Symptomfreiheit erzielt wird. Hier ist weitere Forschung notwendig, um die Moderatoren und Mediatoren für Therapieerfolg bzw. -misserfolg herauszufinden.

Für die Anorexia nervosa liegt begrenzte Evidenz dafür vor, dass eine speziell auf die Erkrankung ausgerichtete Psychotherapie einem unspezifischen psychotherapeutischen Vorgehen vorzuziehen ist. Allerdings ergaben sich bei der Behandlung der Anorexia nervosa bisher keine Überlegenheiten für spezielle psychotherapeutische Verfahren.

Bei der Binge-Eating-Störung liegen die meisten Wirksamkeitsbelege für die kognitiv-behaviorale Therapie vor. Aber auch die interpersonelle Therapie und die manualisierte Selbsthilfe zeigen gute Ergebnisse. Ein großes Problem ist allerdings über alle Behandlungsansätze hinweg die Reduktion des Übergewichtes bzw. der Adipositas. Hier zeigen sich bisher keine nachhaltigen Befunde.

Frau Professor Tuschen-Caffier, ich danke Ihnen für dieses Gespräch.

\section{Literatur}

Hay PP, Bacaltchuk J, Stefano S, Kashyap P: Psychological treatments for bulimia nervosa and binging. Cochrane Database Syst Rev 2009;7(4):CD000562. 